\title{
Germanica
}

35 | 2004

La figure de l'imposteur dans la littérature de langue allemande au $\mathrm{xx}^{\circ}$ siècle

\section{Figures d'imposteurs chez Friedrich Dürrenmatt et Peter Weiss : de la double imposture dans le théâtre contemporain (le théâtre dans le théâtre)}

Hochstaplerfiguren bei Friedrich Dürrenmatt und Peter Weiss : von der

Verdoppelung der Hochstaplerfigur im gegenwartigen Theater

Philippe Wellnitz

\section{(2) OpenEdition}

Journals

Édition électronique

URL : http://journals.openedition.org/germanica/1782

DOI : 10.4000/germanica. 1782

ISSN : 2107-0784

Éditeur

Université de Lille

Édition imprimée

Date de publication : 31 décembre 2004

Pagination : $39-50$

ISBN : 9782913857148

ISSN : 0984-2632

Référence électronique

Philippe Wellnitz, «Figures d'imposteurs chez Friedrich Dürrenmatt et Peter Weiss : de la double imposture dans le théâtre contemporain (le théâtre dans le théâtre) », Germanica [En ligne], 35 | 2004, mis en ligne le 05 octobre 2012, consulté le 06 octobre 2020. URL : http://journals.openedition.org/ germanica/1782 ; DOI : https://doi.org/10.4000/germanica.1782

Ce document a été généré automatiquement le 6 octobre 2020.

(c) Tous droits réservés 


\title{
Figures d'imposteurs chez Friedrich Dürrenmatt et Peter Weiss : de la double imposture dans le théâtre contemporain (le théâtre dans le théâtre)
}

\author{
Hochstaplerfiguren bei Friedrich Dürrenmatt und Peter Weiss : von der \\ Verdoppelung der Hochstaplerfigur im gegenwartigen Theater
}

Philippe Wellnitz

1 Parler de l'imposteur au théâtre semble relever d'une double évidence. En effet, d'un point de vue de l'histoire du théâtre, l'imposteur est un personnage de comédie par excellence : en parcourant le théatre comique européen, on peinerait à en dresser une liste exhaustive. Citons à titre d'exemple Tartuffe (1669) de Molière, Le Réviseur (1836) de Gogol, Knock (1923) de Jules Romains et Der Hauptmann von Köpenick (1930) de Carl Zuckmayer, qui figurent parmi les pièces les plus connues où l'imposteur tient un rôle central.

2 D'autre part on peut aussi appréhender l'imposteur au théâtre non pas comme une figure ou comme un thème, mais, dans un sens plus abstrait, comme un élément constitutif du théâtre tout court. Pas de théâtre sans acteur sur scène. Or, au contraire du personnage romanesque qui assume comme véritables ses pensées et ses paroles (ne serait-ce qu'à travers le truchement d'un narrateur), l'acteur de théâtre joue explicitement le rôle d'un personnage, le personnage est représenté par l'acteur. Tout en simulant les dires et les gestes d'un personnage, l'acteur est néanmoins autre. Pour Anne Ubersfeld, le comédien « est le lieu de tous les paradoxes : il est là et il convoque un personnage absent, il est maître de la parole-mensonge et on lui demande d'être "sincère" ${ }^{1}$.» Il existe de ce fait un pacte tacite entre l'acteur et le spectateur qui est conscient de l'imposture de l'acteur : « La caractéristique de la communication théâtrale, c'est que le récepteur considère le message comme non réel ou plus exactement comme 
non $\mathrm{vrai}^{2} . »$ En d'autres termes, le théâtre représenté est une forme socialement admise de l'imposture, voire une de ses formes socialisées ${ }^{3}$. On peut en déduire l'hypothèse suivante : l'imposture du jeu théâtral et en particulier l'imposteur au théâtre (l'acteur) assument un rôle social, ils sont nécessaires à la société - leur rôle est de nature cathartique si l'on se réfère au théâtre des anciens. Pour notre part, nous aimerions utiliser ici pour l'imposteur au théâtre (tout acteur jouant un personnage) le concept de "mode ironique ", car l'ironie peut se définir par opposition au mensonge ${ }^{4}$ comme une assertion non-vraie dont tous les interlocuteurs savent qu'elle n'est pas vraie, voire signifie l'exact contraire de l'énoncé ironique au premier degré. Cet imposteur au théâtre qu'est l'acteur s'apparente en effet au locuteur ironique : il ne cache pas, lui non plus, son imposture, elle est même sa raison d'être sur scène. C'est pourquoi l'acteur devenu personnage simulé sur scène fonctionne selon nous sur le "mode ironique ». En cela, il est d'emblée différent des autres figures de l'imposteur dans d'autres genres littéraires, dont l'imposture cherche à s'occulter au niveau intradiégétique et qui est tout au plus dévoilée tardivement par d'autres personnages ou au niveau extradiégétique par le narrateur ou le travail du lecteur. Il y a donc clairement une spécificité du théâtre en ce qui concerne la figure de l'imposteur qui est en quelque sorte un imposteur dédoublé. Parler d'un imposteur dans le cadre d'un texte dramatique signifie alors s'intéresser à une double imposture : celle de l'acteur et celle du personnage incarné.

Dans notre analyse de l'imposteur au théâtre, nous avons opéré un double choix : D'une part nous nous limitons à des exemples du théâtre de langue allemande au $\mathrm{xx}^{\mathrm{e}}$ siècle, d'autre part nous abordons le phénomène particulier du théâtre dans le théâtre pour mieux appréhender l'évolution de la figure de l'imposteur dont nous venons de souligner le dédoublement propre au genre théâtral.

En effet, Romulus der Grosse (1949) de Friedrich Dürrenmatt et Die Verfolgung und Ermordung Jean Paul Marats dargestellt durch die Schauspielgruppe des Hospizes zu Charenton unter Anleitung des Herrn de Sade (1964) de Peter Weiss, mettent en scène des impostures soit explicites sous forme de théâtre dans le théâtre comme dans Marat, soit implicites comme dans Romulus der Grosse dont le protagoniste éponyme joue la comédie à ses sujets.

Dans Romulus der Grosse de Friedrich Dürrenmatt, il s'agit du dernier empereur romain qui, apparemment impassible, passe son temps à élever des poules dans sa villa de Campanie, alors que l'invasion des armées germaniques avance à grands pas et que ses compatriotes et ses proches semblent désespérer de la situation. Le protagoniste qui donne son nom à la pièce Romulus der Grosse incarne en effet à nos yeux la figure de l'imposteur, puisque Romulus arbore un masque d'incapable face aux autres personnages ignorant son vraie rôle (qui se situe, comme nous allons le voir, aux antipodes de sa fonction officielle qui est de représenter et de défendre l'Empire romain).

Cet empire et son premier représentant apparaissent dans un premier temps sous une lumière comique : déjà le titre contient une coloration comique par l'allusion parodique au fondateur de Rome et par la contradiction entre le suffixe diminutif du nom et son titre de "grand». Ainsi, le spectateur est averti d'emblée que la grandeur de ce personnage n'est que factice - tout comme l'est l'Histoire telle qu'elle sera présentée dans la pièce : le sous-titre de la pièce («Eine ungeschichtliche historische Komödie in vier Akten ») contient également une contradiction qui vise à inscrire le propos en 
dehors de la vérité historique strictu sensu. L'exergue choisie par l'auteur, une citation de Lichtenberg ${ }^{5}$, insiste sur la nécessité de s'écarter de la stricte vérité pour atteindre l'effet comique.

7 Bien des éléments nous indiquent en tout cas dès le départ qu'il s'agit d'une vision satirique de l'Histoire : Le côté artificiel et désuet de cette gloire d'Etat est représenté chez Dürrenmatt dès les premières didascalies par les bustes des empereurs romains qui se trouvent dans des pièces délabrées du siège d'été de Romulus qu'il habite aussi en hiver et où il ne reste que quelques chaises branlantes. Seuls «Pyrame» et "Achille ", deux serviteurs figés comme des statues de marbre, incarnent encore cette gloire passée qui ne permet aucune entorse au cérémonial ${ }^{6}$ et au rôle éminent qui est le leur : "Wer unseren Wert verkennt, schaufelt Rom das Grab7. " (16) L'Empereur luimême semble avoir une vision bien peu traditionnelle de la hiérarchie, car pour Romulus, l'homme le plus important de son Empire, c'est son cuisinier (17) - dont on mesure l'importance dans la parodie du Tu quoque, mi fili, lorsque Romulus le retrouvera parmi les conjurés : «Koch, auch du? » (88). Jusque là, il ne s'agit toutefois que de railleries et de parodies comiques.

8 Mais bien au-delà de ces simples traits comiques, Romulus parait être un empereur qui ne remplit pas bien son rôle tant sa nonchalance semble confiner à la paresse. Cela pose cet empereur comme imposteur au premier degré qui usurperait un pouvoir dont il ne serait pas digne. Effectivement, Romulus éconduit son Ministre de l'intérieur Tullius Rotundus qui lui fait part de l'arrivée du messager Spurius Titus Mamma lequel veut annoncer la chute de Pavie. Romulus refuse de le recevoir avant que celui-ci ne se soit reposé : «Auch die schlimmste Meldung klingt aus dem Munde eines wohlausgeruhten, frisch gebadeten und rasierten Menschen, der gut gegessen hat, noch ganz angenehm. Er soll morgen kommen.» (21). Romulus considère que les nouvelles ne chamboulent guère le monde car elles ne rapportent que ce qui s'est déjà passé et ce qui ne peut plus être changé. Même l'impératrice et le Ministre de la guerre n'arrivent pas à le convaincre d'entendre le messager. Cependant, Romulus qui a donné à chacune de ses poules qu'il élève le nom d'un empereur romain ou d'un chef germanique, apprend par son serviteur Pyrame que (la poule) Odoacre a pondu trois œufs, un record, et conclut : « Ich brauche Spurius Titus Mamma nicht mehr. Der Germanenfürst Odoaker hat Pavia erobert, denn das Huhn seines Namens hat drei Eier gelegt.» (27) Exhorté à agir, Romulus propose de rédiger une proclamation aux troupes et nomme son Ministre de la guerre au rang de Maréchal d'empire. L'impératrice lui dit que cela ne sert à rien et Romulus lui répond : «Du kannst doch unmöglich von mir verlangen, daß ich mehr tue als regieren, liebe Frau. » (28). Lorsque sa femme demande à Romulus de lui faire enfin part de ses propres projets, il dit simplement : "Ich möchte die Weltgeschichte nicht stören, liebe Julia. » (45).

On voit bien à quel point le comique du personnage rend l'exercice de sa fonction peu crédible, le mue en imposteur aux yeux des autres.

Mais cette critique de l'imposteur ne sera pas le seul fait des personnages de la pièce : Lorsque l'empereur byzantin Zénon se réfugie chez Romulus, la seule solution est pour eux de croire à leur rôle respectif face aux destinées de ce monde. Les didascalies condamnent sans appel la vacuité de ce mode de gouverner : "Schweigen. Man sitzt in gläubiger Haltung da. » (34) «Alle drei sitzen gläubig da. » (35). On comprend alors que l'imposture ne se limite pas à un seul personnage mais s'étend à un système dans son ensemble. Cette critique implicite du système se situe hors dialogue, dans les 
didascalies, ce qui souligne les possibilités accrues du genre théâtral et de ses divers langages pour dénoncer toute imposture.

11 Cette impression d'insouciance et d'incompétence de Romulus est largement partagée par tous, si l'on en juge par la réaction acerbe du héros Émilien, fiancé de la fille de Romulus, qui, revenant du champ de bataille, a perdu sa foi en l'homme dans cette guerre dont il a été la victime :

Willkommen, Imperator des guten Essens und des gesegneten Schlafs in der Mittagshitze. Sei gegrü_t, Cäsar der Hühner und Stratege des Eierlegens! Heil dir, den die Soldaten Romulus den Kleinen nennen. [...] Es gab Kaiser, denen rief man zu : Gut gesiegt, Erhabener? Anderen : Gut gemordet, Majestät? Und so wird man dir zurufen : Gut geschlafen, Kaiser Romulus ? (66).

Une solution, digne de la comédie que cette pièce n'est qu'en apparence, se présente alors : un riche marchand de pantalons germanique est disposé à investir une fortune pour soudoyer les troupes germaniques et sauver ainsi l'Empire romain - à condition que les pantalons deviennent obligatoires à Rome et que Romulus lui accorde la main de sa fille, condition avec laquelle même son fiancé Émilien est d'accord. Mais Romulus refuse cette dernière condition, la comédie bascule alors vers la tragédie ou plutôt vers le tragi-comique. Le troisième acte, qui se déroule aux ides de mars, sera la nuit où l'on tentera de se débarrasser de cet empereur-imposteur apparemment trop près de son confort personnel.

Avant de le quitter pour prendre la fuite, l'impératrice Julia invoquera d'abord leur amour puis le sens du devoir pour que Romulus accepte de marier sa fille pour sauver l'Empire romain. Romulus déclare qu'une résistance à tout prix serait ce qu'il y a de plus insensé et qu'un monde perdu ne doit être mis à feu et à sang. Là encore, Romulus fait figure de poltron aux yeux des autres : «Du hast in den zwanzig Jahren deiner Regierung nichts anderes getan als gegessen, getrunken, geschlafen, gelesen und Hühner gezüchtet. [...] Hinter dir steht nichts als deine Faulheit. » (76).

Mais c'est à cet instant que Romulus s'arrache lui-même son propre masque d'imposteur. Ce détail a son importance: Si l'imposteur «classique » au théâtre est souvent démasqué malgré lui, ce n'est pas le cas ici. On comprend alors que l'on quitte subrepticement l'univers de la simple comédie (de mœurs) pour un autre genre, celui de la satire. Autrement dit, l'imposture de Romulus, qui consistait à exercer ses fonctions en tant qu'empereur indigne, avait un sens supérieur, l'imposture première en cachait une autre, plus insidieuse. Accusé, Romulus devient accusateur.

Romulus avoue qu'il n'a jamais aimé sa femme, que son seul but était de devenir empereur grâce à elle, fille illégitime du précédant empereur qui avait besoin d'un mari noble pour la légitimer et devenir impératrice. Romulus révèle en effet : « Ich bin allein aus politischer Einsicht Kaiser geworden. [...] Es ist meine politische Einsicht, nichts zu tun. » (76). L'impératrice lui rétorque qu'il n'aurait pas eu besoin de devenir empereur pour autant, et Romulus réplique : «Nur so konnte natürlich mein Nichtstun einen Sinn haben. Als Privatmann zu faulenzen ist völlig wirkungslos. » (76) Lorsque Julia dit que cette paresse menace l'État romain, Romulus avoue que c'était bien là son but secret :

Ich bezweifle nicht die Notwendigkeit des Staates, ich bezweifle nur die Notwendigkeit unseres Staates. Er ist ein Weltreich geworden und damit eine Einrichtung, die öffentlich Mord, Plünderung, Unterdrückung und Brandschatzung auf Kosten der andern Völker betrieb, bis ich gekommen bin. [...] Das römische Weltreich besteht seit Jahrhunderten nur noch, weil es einen Kaiser gibt. Es blieb 
mir deshalb keine andere Möglichkeit, als selbst Kaiser zu werden, um das Imperium liquidieren zu können. (77) 
bien sûr toutes les techniques de distanciation propres au théâtre épique de Brecht, les modes langagiers caractéristiques du théâtre absurde pour ne citer que deux exemples.

En l'occurrence, nous allons nous intéresser au topos du théâtre dans le théâtre qui quoique associé à juste titre d'emblée au Théâtre baroque ${ }^{9}$, à une époque où l' ordo était un des fondements spirituels de ce théâtre dans les pays catholiques, - semble s'imposer comme par lui-même au théâtre engagé du vingtième siècle : Le théâtre dans le théâtre constitue à nos yeux une réponse esthétique plus forte à la difficulté de lutter contre la duplicité politique. En effet, si on a à l'esprit le caractère de double imposture que nous pensons déceler dans toute représentation de l'imposteur au théâtre, cette réponse, chez Dürrenmatt du moins, s'est avérée insuffisante. Le théâtre dans le théâtre serait alors une potentialisation de l'imposture théâtrale qui s'opposerait à l'imposture du Pouvoir.

Georges Forestier ${ }^{10}$ a développé quatre grandes significations de ce procédé d'enchâssement «auxquelles la notion de miroir sert de dénominateur commun ${ }^{11}$. Il écrit :

Le théâtre dans le théâtre, c'est toujours le théâtre qui se dédouble. Il y a d'une part le miroir réfléchissant qui renvoie au public l'image du monde du théâtre. Il y a ensuite le miroir trompeur qui joue sur les ressemblances, fait hésiter entre la réalité et son double. [...] Il y a d'autre part le miroir oblique, ou miroir convexe, ni reproduction, ni illusion, mais révélation: le théâtre se dédouble pour instruire le spectateur. Leçon religieuse, leçon humaniste, leçon politique ou morale, ces trois types de révélation cultivés dans la première moitié du [xviie] siècle laisseront vite la place à la "révélation interne » où la pièce intérieure ne renvoie plus qu'au spectacle lui-même ${ }^{12}$.

Dans un contexte politique et esthétique totalement différent, Peter Weiss a choisi de recourir à cet ancien procédé de miroir, au théâtre dans le théâtre, pour sa pièce de 1964 Die Verfolgung und Ermordung Jean Paul Marats dargestellt durch die Schauspielgruppe des Hospizes zu Charenton unter Anleitung des Herrn de Sade.

Il s'agit d'une pièce qui se déroule en 1808 , qui a donc pour cadre temporel les années post-révolutionnaires où domine l'esprit de restauration napoléonienne. Le lieu de l'action est un asile d'aliénés où l'on joue une pièce qui a pour sujet l'assassinat de Marat par Charlotte Corday en 1793. Cette pièce est montée et dirigée par une des personnes internées, le Marquis de Sade qui fait revivre son propre passé par cette mise en scène. Il apparaît rapidement que les personnes internées, au premier chef le Marquis de Sade ${ }^{13}$, ne sont pas toutes aliénées, que bien des personnes se trouvent enfermées dans cet asile de fous pour des raisons politiques.

Le présentateur, qui évoque bien sûr d'abord Marat et sa meurtrière Charlotte Corday, citera en troisième lieu le personnage incarnant Duperret «interniert von wegen politischer Radikalität » (15) ainsi que le personnage du prêtre révolutionnaire Jacques Roux qui ne peut être joué tel qu'il était en réalité car : « Leider hat die Zensur sehr viel / gestrichen von seinen Aussagen im Spiel / denn sie gingen in ihrem Ton zu weit / für die Ordnungsbewahrer in unserer ${ }^{14}$ Zeit $»(16)^{15}$.

On voit bien que le jeu se fait sous contrôle politique et que l'orientation voulue par Coulmier, le directeur de cet asile, est une représentation « zu Ihrer Unterhaltung und zur Erbauung der Kranken » (12) - «Schließlich sind heute andere Zeiten als damals / und wir sollten uns bemühen / die längst überwundenen Mißstände / in einem etwas verklärten Schimmer zu sehen.»(23). Ces propos lénifiants du directeur se trouvent bien sûr dans la pièce-cadre et tout l'intérêt de l'étude de la pièce enchâssée et de son 
rapport à la pièce-cadre est de savoir si elle obéira, plus précisément si le Marquis de Sade obéira, à ces directives politiques ou si sous le masque de metteur en scène d'une distraction édifiante, Sade n'est pas un imposteur, à savoir un révolutionnaire sous cape.

En effet, le chœur mis en scène par Sade intervient de manière ambiguë : « Marat was ist aus unserer Revolution geworden / Marat wir wolln nicht mehr warten bis morgen / Marat wir sind immer noch arme Leute / und die versprochenen Änderungen wollen wir heute.»(21). On hésite lorsqu'il s'agit de savoir si ces propos s'adressent uniquement aux personnages de la pièce enchâssée (qui se déroule en 1793) ou s'ils ne s'adressent pas en même temps aux acteurs (de la pièce / de la révolution), autrement dit au niveau temporel des personnes qui sont spectateurs de cette représentation (en 1808). Ce «miroir trompeur qui joue sur les ressemblances, fait hésiter entre la réalité et son double ${ }^{16}$, écrit Forestier. Le personnage de Sade navigue d'ailleurs allègrement entre la pièce-cadre et la pièce enchâssée puisqu'il la met en scène et y participe dans son propre rôle lorsqu'il débat avec Marat. L'imposture des acteurs jouant un rôle chercherait donc à se détruire elle-même pour rejaillir sur les spectateurs de ce jeu qui peuplent la pièce-cadre. Mutatis mutandis, l'illusion de la scène s'estompe pour un effet de miroir convexe: si le théâtre dans le théâtre n'est qu'un procédé d'imposture qui s'avoue tel, si ce double jeu sert à démasquer la prétendue réalité, alors seule l'imposture scénique est en liaison avec la réalité, elle seule dit vrai. C'est en tout cas le point de vue défendu par Sade qui juge toute activité politique vaine : «Was wir tun ist nur ein Traumbild / von dem was wir tun wollen / und nie sind andere Wahrheiten zu finden / als die veränderlichen Wahrheiten der eigenen / Erfahrungen» (45) - Sade s'adressera à Marat ainsi : "Du wolltest dich einmengen in die Wirklichkeit / und sie hat dich in die Enge gedrängt / Ich / habe es aufgegeben mich mit ihr zu befassen / mein Leben ist die Imagination / Die Revolution / interessiert mich nicht mehr» (48). L'imagination, le rêve ou le jeu sur scène, touchent plus à la vérité que l'action dans la vie réelle, semble dire Sade. En affirmant cela, on n'est pas loin d'évoquer la traditionnelle idée du theatrum mundi, si ce n'est qu'aux yeux du théâtre du vingtième siècle, la vie n'est plus un songe ou une parabole de la vanité humaine, mais bien le champ de bataille d'affrontements sociaux qui se cachent sous le masque de l'imposture d'honorabilité. En tant que spectateurs que nous sommes, cela signifie que le procédé de double imposture - des acteurs nous jouent des personnages qui à leur tour endossent le rôle d'acteurs jouant des personnages - nous livre en quelque sorte un mode d'emploi pour cette pièce de Peter Weiss afin de comprendre notre époque, dans laquelle nous jouons tous un rôle. En effet, l'annonceur dit bien à la fin du premier acte qu'en jouant il va présenter un jeu simulée ${ }^{17}$, donc en fait ce jeu est bien un miroir de la réalité.

C'est en fait ce rôle de miroir que Sade incarne dans cette pièce : Par sa pièce enchâssée qui prend littéralement le pas sur la réalité de la pièce-cadre, il détruit à la fois l'imposture de l'illusion théâtrale et l'illusion des imposteurs de réalité. Marat a beau proclamer «Ich bin die Revolution » (27), il n'est en réalité qu'un imposteur qui avait échoué face à la réalité déjà dans d'autres rôles usurpés ${ }^{18}$. C'est la fin de la mise en scène de Sade qui déclenche la vraie révolution, lorsque jeu et réalité, pièce enchâssée et pièce-cadre se confondent en une seule scène: Le personnage Jacques Roux, imposteur caché sous une soutane ${ }^{19}$, va établir dans la dernière réplique de la pièce de Peter Weiss le lien entre les trois niveaux de la pièce, entre personnages, acteurs, 
spectateurs internes de la pièce-cadre et spectateurs externes que nous sommes: "Wann werdet ihr endlich sehen lernen / Wann werdet ihr endlich verstehen » (136).

Le théâtre dans le théâtre aurait alors une fonction bien particulière : celle de briser l'imposture socialement admise du jeu théâtral, une imposture qui jusqu'alors lui ôtait tout son potentiel révolutionnaire, comme on l'a vu chez Dürrenmatt. Par ce biais d'imposture théâtrale brisée, toute imposture socialement admise (le théâtre comme jeu simulé, mais aussi toutes les autres simulations de notre société) serait détruite. On commencerait alors à voir dans le théâtre libéré de ses chaînes de l'imposture artistique un miroir de la réalité. En effet, si l'acteur de la pièce enchâssée abandonne l'imposture du rôle de son personnage au profit d'un effet de "miroir oblique, ou miroir convexe ", il n'y aurait « ni reproduction, ni illusion, mais révélation : le théâtre se dédouble[rait] pour instruire le spectateur ${ }^{20}$.

Nous venons d'établir un lien entre d'une part l'illusion théâtrale brisée par son dédoublement dans le théâtre et la fin de l'imposture sociale d'autre part. Autrement dit, le théâtre de Peter Weiss espère retransformer l'acteur devenu personnage en acteur qui agit non plus sur scène mais avec la scène sur nous. Ces imposteurs modernes que l'on trouve dans le théâtre du vingtième siècle ont pour but (finalement) avoué d'agir à l'opposé de l'imposteur classique qui fait tout pour ne pas être découvert. Détruire l'imposture du jeu théâtral, démasquer l'imposteur au théâtre à l'aide de l'imposteur lui-même, c'est aussi un peu détruire l'imposteur qui sommeille dans tout un chacun. En cela et par des choix esthétiques et politiques différents, Friedrich Dürrenmatt et Peter Weiss ont renouvelé, à partir du personnage-type qu'est l'imposteur, tout notre rapport à la représentation théâtrale.

\section{NOTES}

1. Anne UBERSFELD Lire le théâtre II (L'École du spectateur), Paris, Belin, 1996 (1977), p. 137.

2. Anne UBERSFELD Lire le théâtre I, Paris, Belin, 1996 (1977), p. 35.

3. On peut très bien imaginer d'autres formes d'imposture admises par la société : toutes les règles de politesse et leurs verbalisations par exemple. D'une manière générale, tout acte symbolique ou rituel contient une part d'imposture puisqu'il est admis qu'il signifie autre chose que ce qu'il signale au premier degré.

4. C'est ainsi qu'elle est définie dans le volume collectif de Catherine KERBRAT- ORECCHIONI et alii (dir.) L'ironie, Presses Universitaires de Lyon, 1978.

5. «Der große Kunstgriff, kleine Abweichungen von der Wahrheit für die Wahrheit selbst zu halten, worauf die ganze Differentialrechnung gebaut ist, ist auch zugleich der Grund unserer witzigen Gedanken, wo oft das Ganze hinfallen würde, wenn wir die Abweichungen in einer philosophischen Strenge nehmen würden. »

6. Ces doubles sont à leur tour dédoublés par les deux serviteurs de Zénon, encore plus formalistes que les premiers. Ce dédoublement comique des serviteurs et de leurs propos est un procédé que Dürrenmatt réemploiera pour sa pièce à succès Der Besuch der Alten Dame de 1956. 
7. Nous citons Romulus der Große d'après l'édition des œuvres de Friedrich Dürrenmatt en 30 volumes (Werkausgabe) de 1980, Die Verfolgung und Ermordung Jean Paul Marats dargestellt durch die Schauspielgruppe des Hospizes zu Charenton unter Anleitung des Herrn de Sade de Peter Weiss d'après l'édition suhrkamp [es 68] de 1964/65 et Achterloo de Friedrich Dürrenmatt d'après sa traduction française parue en 1989 chez l'Âge d'Homme - en incluant respectivement l'indication de la page dans le corps du texte.

8. Sans que vraisemblablement Albert Camus ne l'ait connu, il y a dans ce personnage de Romulus, imposteur-qui-démasque, quelque chose qui annonce le «juge-pénitent " Clamence dans sa nouvelle La Chute (1956) : «Je m'accuse en long et en large. [...] Mais du même coup, le portrait que je tends à mes contemporains devient un miroir. [...] Alors, insensiblement, je passe, dans mon discours, du « je » au «nous ». [...] Je suis comme eux, bien sûr, nous sommes dans le même bouillon. J'ai cependant une supériorité, celle de le savoir, qui me donne le droit de parler. [...] Plus je m'accuse et plus j'ai le droit de vous juger. » [Paris, éd. folio, pp. 147/148].

9. À titre d'exemple pour ces pièces du XVII ${ }^{\mathrm{e}}$ siècle, on peut citer Le Grand Théâtre du Monde de Calderón, L'illusion comique de Corneille, Peter Squentz de Gryphius, Hamlet, Songe d'une Nuit d'été et La Tempête de Shakespeare.

10. Georges FORESTIER, Le Théâtre dans le Théâtre, Paris, Droz, 1996.

11. Georges FORESTIER, Le Théâtre dans le Théâtre, p. 16.

12. Ibidem.

13. Sade, « der unter eines anrüchigen Ruhmes Stern / seit fünf Jahren in unsrer Anstalt weilt / von zahlreichen Verfolgungen und Prüfungen ereilt / [...] / Autor von Werken die verkannt und verbrannt / und um derentwillen man ihn Jahrzehnte verbannt » (17).

14. C'est nous qui soulignons ce terme.

15. Nous marquons les sauts de lignes par des barres obliques en raison des rîmes contenues dans ces répliques.

16. Georges FORESTIER, Le Théâtre dans le Théâtre, p. 16.

17. «als würde hier zum Schein / nur vorgetragen und gespielt » (96).

18. «Und als sie herausfanden / daß er nur ein Kurpfuscher war / der seine Medizinen aus Wasser und Kreide herstellte / und als sie ihn auf die Gasse hinauswarfen / auf der er zuhause war / da begann er zu schrein /[...] / Besitz ist Diebstahl / und / Nieder mit den Tyrannen » (91); «Und als er es mit seiner Forscherei zu nichts brachte / [...] / Da kam diesem Dilettanten die Revolution gerade gelegen / [...] / Und er ging zu den Unterdrückten über / [...] / Und nannte sich Freund des Volkes / [...] / Doch er dachte nicht an das Volk / [...] / Sondern nur an seine eigene Unterdrücktheit » (94).

19. Sade encourage cette imposture sur scène: «Bravo Jacques Roux / Deine mönchische Kleidung ist nach meinem Sinn / denn das ist jetzt [impossible de ne pas remarquer l'ambiguité qui plane sur ce déictique temporel] das beste / immer bereit zu sein / sich im Sack zu verkriechen / aufzutauchen in günstigen Momenten / dann schnell wieder weg wenn's sein muß / Deine Kutte Jacques Roux / ist ein gutes Mimikri » (66/67).

20. Georges FORESTIER, Le Théâtre dans le Théâtre, p. 16. 


\section{RÉSUMÉS}

L'imposteur au théâtre a non seulement une longue tradition (Tartuffe, Le Réviseur, Knock, etc.), il a surtout une particularité par rapport aux autres figures littéraires de l'imposteur dans les textes en prose: il existe un accord tacite entre le spectateur et l'acteur qui feint d'être un personnage, le théâtre en tant que jeu est une forme socialisée de l'imposture.

Chez Friedrich Dürrenmatt et Peter Weiss, il y a un dédoublement supplémentaire de l'imposteur. Dans Romulus der Grosse (1949), le protagoniste s'arrache lui-même le masque de l'imposteur candide pour révéler une autre imposture, plus subtile, qui répond à l'imposture de l'État et de l'Histoire. Dans Die Verfolgung und Ermordung Jean Paul Marats dargestellt durch die Schauspielgruppe des Hospizes zu Charenton unter Anleitung des Herrn de Sade (1964), il y a d'abord un dédoublement feint par le procédé du théâtre dans le théâtre. Mais Peter Weiss abolit rapidement les limites qui séparent la pièce enchâssée de la pièce-cadre. Ainsi, c'est l'abolition de l'imposture théâtrale en général, le basculement du jeu vers la réalité, qui devrait entraîner l'abolition de toute imposture socialement admise jusqu'alors.

Ces imposteurs du théâtre moderne qui, à l'opposé, de l'imposteur classique se démasquent euxmêmes ne sont plus des accusés, mais des accusateurs.

Hochstaplerfiguren im Drama haben nicht nur eine lange theatergeschichtliche Tradition (Tartuffe, Der Revisor, Der Hauptmann von Köpenick, u.v.a.), sondern es herrscht auch im Gegensatz zu solchen Gestalten in der Prosa ein stillschweigendes Übereinkommen zwischen Publikum und Schauspieler, der eine Figur spielt. Anders gesagt ist Theater eine sozialisierte Form der Hochstapelei.

Im Theater von Friedrich Dürrenmatt und von Peter Weiss gibt es eine weitere Verdoppelung der Hochstaplerfigur : in Romulus der Grosse entlarvt sich der hochstaplerische Kaiser selbst, um die Hochstapeleien des Vaterlands anzuprangern; in Marat gibt es ein Spiel im Spiel, bei dem die Grenzen zwischen Rahmen-und Binnenstück sich langsam verwischen: dadurch wird die sozialisierte Hochstapelei des Bühnenspiels allgemein aufgehoben, das Umkippen des Spiels in die Wirklichkeit soll aber auch alle anderen sozialisierten Formen der Hochstapelei ins Wanken bringen. Diese Hochstapler des modernen Dramas reissen sich im Gegensatz zu klassischen Hochstaplerfiguren selbst die Maske vom Gesicht, sie spielen nicht mehr die Rolle des Angeklagten, sondern die des Anklägers.

\section{AUTEUR}

\section{PHILIPPE WELLNITZ}

Université Paul Valéry-Montpellier 